\title{
The relationship between monocyte-to-lymphocyte ratio and the risk of gastrointestinal system involvement in children with IgA vasculitis: A preliminary report
}

\author{
Yuan Yuan ${ }^{1, A-F}$, Jun Liu ${ }^{1, A-F}$, Yongxin Zhou $u^{1, B, E, F}$, Xufang Du $u^{1, B, C, E, F}$, Qian Chen ${ }^{1, B, E, F}$, Jiongying Zhou ${ }^{1, C-F}$, Miao Hou ${ }^{2, A-F}$ \\ ${ }^{1}$ Department of Pediatrics, Changshu No. 2 People's Hospital, Jiangsu, China \\ ${ }^{2}$ Department of Cardiology, Children's Hospital of Soochow University, Suzhou, China \\ A - research concept and design; $\mathrm{B}$ - collection and/or assembly of data; $\mathrm{C}$ - data analysis and interpretation; \\ $\mathrm{D}$ - writing the article; $\mathrm{E}$ - critical revision of the article; $\mathrm{F}$ - final approval of the article
}

Address for correspondence

Miao Hou

E-mail: houmia0321@126.com

Funding sources

None declared

Conflict of interest

None declared

Received on February 10, 2021

Reviewed on May 5, 2021

Accepted on June 14, 2021

Published online on September 6, 2021

Cite as

Yuan Y, Liu J, Zhou Y, et al. The relationship between monocyte-to-lymphocyte ratio and the risk

of gastrointestinal system involvement in children with

IgA vasculitis: A preliminary report. Adv Clin Exp Med.

2021;30(10):999-1005. doi:10.17219/acem/138906

DOI

10.17219/acem/138906

Copyright

Copyright by Author(s)

This is an article distributed under the terms of the

Creative Commons Attribution 3.0 Unported (CC BY 3.0)

(https://creativecommons.org/licenses/by/3.0/)

\begin{abstract}
Background. Immunoglobulin A ( $\lg A)$ vasculitis is the most common systemic vasculitis of childhood. It can affect the gastrointestinal system (GS) and the renal system.

Objectives. To evaluate the monocyte-to-lymphocyte ratio (MLR) and other hematological markers in predicting GS and renal system complications of IgA vasculitis in children.

Materials and methods. One hundred and fifteen children with IgA vasculitis and 95 healthy children were included in this study. Demographic characteristics, organ involvement, and laboratory findings, including neutrophil, lymphocyte, monocyte and platelet (PIt) counts, red blood cell volume distribution width (RDW), platelet distribution width (PDW), mean platelet volumes (MPV), monocyte/platelet counts, neutrophil/ lymphocyte ratio (NLR), and platelet/lymphocyte ratio (PLR), were evaluated.

Results. Among 115 children with IgA vasculitis, 34 (29.5\%) cases had GS involvement, and renal involvement was observed in 12 children (10.4\%). Neutrophil, monocyte and Plt count and MLR, NLR and PLR values were higher in the IgA vasculitis group than in control groups. Moreover, the neutrophil count and NLR and MLR levels were significantly higher in children with GS involvement than in those without GS involvement. Logistic regression analysis showed MLR was the sole risk factor for GS involvement among these parameters. Furthermore, a cut-off MLR value of 0.245 differentiated children with lgA vasculitis with GS involvement from those without GS involvement (area under the curve (AUC) 0.694, with a sensitivity of $52.9 \%$ and specificity of $77.8 \%$ ).
\end{abstract}

Conclusions. An elevated MLR value could serve as a useful marker in predicting GS involvement in IgA vasculitis in children. Therefore, monitoring the blood MLR value may serve as an important novel indicator to pediatricians regarding the involvement of GS and disease severity of IgA vasculitis.

Key words: IgA vasculitis, neutrophil-to-lymphocyte ratio, gastrointestinal system involvement, monocyteto-lymphocyte ratio, platelet-to-lymphocyte ratio 


\section{Background}

Immunoglobulin A (IgA) vasculitis is the most common systemic vasculitis of childhood. ${ }^{1}$ The average annual incidence of this disease in children is about $20 / 100,000{ }^{2}$ Although the disease is most often characterized by a mild, self-limiting course with a good prognosis, it may present a progressive course and high recurrence in some cases. ${ }^{3}$ It not only affects the small vessels of the skin, but also the gastrointestinal and renal systems in some cases, including severe gastrointestinal bleeding and renal insufficiency. ${ }^{4}$ Therefore, easily accessible and cost-effective laboratory parameters that can be used to predict the severity of the disease are needed.

Blood cell-derived parameters, including white blood cell (WBC) and its subtype cell counts (neutrophils, lymphocytes and monocytes), hemoglobin ( $\mathrm{Hb})$, red blood cell volume distribution width (RDW), platelet (Plt), platelet distribution width (PDW) and mean platelet volume (MPV), are commonly used clinical indicators, ${ }^{5-7}$ which also have the advantage of simple retrieval from routine blood examination, with established detection methods and rapid results. Moreover, neutrophil-to-lymphocyte ratio (NLR) and platelet-to-lymphocyte ratio (PLR) are new inflammatory indicators easily obtained from blood count. Recently, studies have shown that NLR and PLR are increased in a variety of other conditions, including cardiovascular disease, ${ }^{8}$ diabetes mellitus, ${ }^{9}$ immune system diseases, ${ }^{10}$ and cancer, ${ }^{11}$ and they can be used as novel inflammation parameters to predict the severity of these diseases. Similarly, NLR and PLR were reported to be associated with gastrointestinal bleeding among children with recent IgA vasculitis. ${ }^{12}$ Of note, monocyte-to-lymphocyte ratio (MLR) was also suggested as a new inflammatory marker, and its clinical value has been investigated in patients with cancer, ${ }^{13}$ cirrhosis ${ }^{14}$ and retinopathy. ${ }^{15}$ However, few studies have investigated the relationship between MLR and severity of IgA vasculitis in children.

\section{Objectives}

We aimed to study the value of MLR and other blood cell-derived parameters, including neutrophil, lymphocyte, monocytes and Plt count, MPV, NLR and PLR in predicting the disease course, including gastrointestinal and renal involvement and recurrence in children with IgA vasculitis.

\section{Materials and methods}

\section{Study design and participants}

This study was conducted in full accordance with the Declaration of Helsinki and was approved by the Ethics
Committee of the Changshu No. 2 People's Hospital (approval No. 2020-KY-45).

This retrospective study included 115 children with IgA vasculitis who were admitted to the Department of Pediatrics, Changshu No. 2 People's Hospital from January 1, 2016 to January 1, 2020. The IgA vasculitis was diagnosed according to the EULAR/PRINTO/PRES 2013 criteria. ${ }^{16,17}$ Ninety-five age and gender-matched healthy children were enrolled as the control group. Patients with other autoimmune diseases; severe heart, lung and endocrinal diseases; or those with fungal and tuberculosis infections were excluded.

Gastrointestinal system (GS) involvement was defined as the presence of abdominal pain, vomiting or gastrointestinal hemorrhage. ${ }^{18}$ Renal involvement was defined as the presence of hematuria ( $>5$ red blood cells per high-power microscopic field) and/or proteinuria (protein concentration in spot urine $\geq 30 \mathrm{mg} / \mathrm{dL}$ or spot urine protein/creatinine ratio $>0.5$ in children $<2$ years of age and $>0.2$ in children $\geq 2$ years of age). ${ }^{19}$ The recurrence of IgA vasculitis was defined as a new flare of cutaneous lesions or other manifestations of vasculitis in a patient at least 4 weeks after the initial IgA vasculitis diagnosis. ${ }^{3}$

\section{Laboratory assessments}

The venous blood sample was collected in the fasting state at the time of admission to the hospital (before the start of the treatment). Blood routine, urine routine and fecal occult blood tests were conducted by the Department of Clinical Laboratory of Changshu No. 2 People's Hospital. The leukocyte count (neutrophil, monocyte and lymphocyte count), Plt count, RDW, Hb, PDW, Creactive protein (CRP), and erythrocyte sedimentation rate (ESR) were recorded, and the NLR, MLR, and PLR were calculated.

\section{Statistical analyses}

All data were analyzed using the SPSS v. 19.0 software package (IBM Corp., Armonk, USA). The ShapiroWilk test was used to test the distribution of analyzed variables. Mean \pm standard deviation $(\mathrm{SD})$ were used to describe continuous data. To compare differences between 2 groups, independent t-tests for continuous variables with normal distribution were used. The percentages were used to describe categorical data, which were analyzed with $\chi^{2}$ tests. Logistic regression analysis was performed for multivariate analysis. The receiver operating characteristic (ROC) curve analysis was conducted to evaluate the cutoff value of blood count parameters. Results with $\mathrm{p}<0.05$ were considered statistically significant. 


\section{Results}

\section{Clinical characteristics and blood cell counts in the IgA vasculitis group and in healthy children}

There were 56 (48.69\%) boys and 59 (51.30\%) girls with IgA vasculitis included in the study. Among the children with IgA vasculitis, 34 (29.56\%) cases had GS involvement, and renal involvement was observed in 12 children (10.43\%); only 3 cases (2.61\%) had both GS and renal involvement. Additionally, 22 (19.13\%) children had at least one recurrence of IgA vasculitis during follow-up.

The WBC, neutrophil, monocyte and Plt counts and MPV, NLR, MLR and PLR values were significantly higher in the IgA vasculitis group compared with control groups. However, there were no differences in lymphocyte count and $\mathrm{Hb}$, RDW and PDW levels between the IgA vasculitis and control groups (Table 1).

\section{Comparison of blood cell count parameters in children with IgA vasculitis with or without internal organ involvement}

The neutrophil count and NLR and MLR levels were found to be significantly higher in children with GS involvement compared to those without GS involvement. However, there was no difference in WBC count, lymphocyte count, monocyte count or Hb, Plt, RDW, PDW, MPV, PLR, CRP and ESR values between the 2 groups (Table 2)
The blood cell parameters were similar between children with and without renal involvement (Table 3). Additionally, there were no differences in hematological parameters between children with IgA vasculitis with or without recurrence groups, except for the PDW value (Table 4).

\section{Multivariate analysis for children with IgA vasculitis with GS involvement}

Independent variables of multivariate analysis were selected as the statistically significant variables between children with IgA vasculitis, with and without GS involvement. The results showed that MLR was an independent risk factor for IgA vasculitis combined with GS ( $<<0.05$, Table 5).

\section{Diagnosis value of MLR in the differential of IgA vasculitis combined with GS involvement}

The ROC curve analysis showed that the area under the curve for MLR diagnosis of IgA vasculitis combined with GS was 0.694 , with a sensitivity of $52.9 \%$ and a specificity of $77.8 \%$, and the cutoff point was 0.245 (Fig. 1).

\section{Discussion}

The IgA vasculitis is an immune complex-mediated small vessel vasculitis with variable skin, gastrointestinal and renal involvement. In order to predict the risk of GS and kidney involvement in IgA vasculitis, several

Table 1. Comparison of clinical characteristics and blood cell-derived parameters between children with immunoglobulin A (lgA) vasculitis and healthy children

\begin{tabular}{|c|c|c|c|c|}
\hline Variable & $\begin{array}{l}\text { IgA vasculitis } \\
\quad(n=115)\end{array}$ & $\begin{array}{l}\text { Control } \\
(n=95)\end{array}$ & Test statistic & $p$-value \\
\hline Age [years] & $8.29 \pm 2.59$ & $8.57 \pm 1.58$ & $-0.90 \dagger$ & 0.3674 \\
\hline Gender (M/F) & $56 / 59$ & $50 / 45$ & $0.32 \neq$ & 0.5702 \\
\hline WBC $\left[10^{9} / L\right]$ & $9.49 \pm 3.62$ & $5.91 \pm 1.24$ & $9.07 \dagger$ & $<0.0001$ \\
\hline$N\left[10^{9} / L\right]$ & $6.08 \pm 3.25$ & $2.93 \pm 0.97$ & $8.97 \dagger$ & $<0.0001$ \\
\hline$M\left[10^{9} / L\right]$ & $0.56 \pm 0.35$ & $0.3 \pm 0.11$ & $7.46+$ & $<0.0001$ \\
\hline$L\left[10^{9} / \mathrm{L}\right]$ & $2.73 \pm 1.32$ & $2.52 \pm 0.54$ & $1.57 \dagger$ & 0.1189 \\
\hline $\mathrm{Hb}[\mathrm{g} / \mathrm{L}]$ & $126.28 \pm 11.26$ & $128.60 \pm 7.21$ & $-1.71+$ & 0.0879 \\
\hline Plt $\left[10^{9} / \mathrm{L}\right]$ & $328.06 \pm 95.94$ & $264.66 \pm 55.89$ & $5.94 \dagger$ & $<0.0001$ \\
\hline RDW & $12.79 \pm 0.9$ & $12.74 \pm 0.58$ & $0.44 \dagger$ & 0.6606 \\
\hline PDW & $13.04 \pm 2.67$ & $12.69 \pm 2.05$ & $1.02+$ & 0.3108 \\
\hline MPV & $10.12 \pm 1.32$ & $10.5 \pm 0.87$ & $-2.37 \dagger$ & 0.0186 \\
\hline NLR & $2.83 \pm 2.41$ & $1.21 \pm 0.47$ & $7.05+$ & $<0.0001$ \\
\hline MLR & $0.22 \pm 0.14$ & $0.12 \pm 0.05$ & $7.31+$ & $<0.0001$ \\
\hline PLR & $147.09 \pm 84.18$ & $110.47 \pm 34.49$ & $4.34 \dagger$ & $<0.0001$ \\
\hline
\end{tabular}

Data are presented as mean \pm standard deviation (SD). P-values were calculated using $X^{2}$ test for categorical variables and the independent t-test for continuous variables. $+\mathrm{t}$-value; $\neq \mathrm{X}^{2}$-value; WBC - white blood cell count; $\mathrm{N}$ - neutrophil count; $\mathrm{M}$ - monocyte count; $\mathrm{L}$ - lymphocyte count; $\mathrm{Hb}$ - hemoglobin; PIt - platelet count; RDW - red blood cell volume distribution width; PDW - platelet distribution width; MPV - mean platelet volumes; NLR - neutrophil-to-lymphocyte ratio; PLR - platelet-to-lymphocyte ratio; MLR - monocyte-to-lymphocyte ratio. 
Table 2. Comparison of clinical characteristics and blood cell-derived parameters between immunoglobulin A (lgA) vasculitis children with gastrointestinal system (GS) involvement and without GS involvement

\begin{tabular}{|c|c|c|c|c|}
\hline Variable & $\begin{array}{l}\text { GS involvement }(+) \\
(\mathrm{n}=34)\end{array}$ & $\begin{array}{l}\text { GS involvement }(-) \\
(n=81)\end{array}$ & Test statistic & $p$-value \\
\hline Age [years] & $8.38 \pm 2.81$ & $8.25 \pm 2.52$ & $0.25+$ & 0.7997 \\
\hline Gender (M/F) & $15 / 19$ & $41 / 40$ & $0.41 \neq$ & 0.5245 \\
\hline WBC $\left[10^{9} / L\right]$ & $10.37 \pm 4.55$ & $9.12 \pm 3.1$ & $1.70+$ & 0.0914 \\
\hline$N\left[10^{9} / L\right]$ & $7.23 \pm 4.02$ & $5.59 \pm 2.76$ & $2.17+$ & 0.0351 \\
\hline$M\left[10^{9} / L\right]$ & $0.66 \pm 0.47$ & $0.51 \pm 0.28$ & $1.77 \dagger$ & 0.0837 \\
\hline$L\left[10^{9} / L\right]$ & $2.37 \pm 1.05$ & $2.88 \pm 1.39$ & $-1.94 \dagger$ & 0.0554 \\
\hline $\mathrm{Hb}[\mathrm{g} / \mathrm{L}]$ & $128.79 \pm 11.19$ & $125.22 \pm 11.18$ & $1.56+$ & 0.1209 \\
\hline Plt $\left[10^{9} / \mathrm{L}\right]$ & $318.38 \pm 103.35$ & $332.12 \pm 93.02$ & $-0.70+$ & 0.4858 \\
\hline RDW & $12.86 \pm 0.94$ & $12.76 \pm 0.89$ & $0.55+$ & 0.5858 \\
\hline PDW & $13.24 \pm 2.71$ & $12.95 \pm 2.66$ & $0.52+$ & 0.6067 \\
\hline MPV & $10.26 \pm 1.37$ & $10.07 \pm 1.31$ & $0.69+$ & 0.4923 \\
\hline NLR & $3.81 \pm 3.25$ & $2.42 \pm 1.83$ & $2.33+$ & 0.0245 \\
\hline MLR & $0.30 \pm 0.18$ & $0.19 \pm 0.11$ & $3.14+$ & 0.0030 \\
\hline PLR & $154.18 \pm 72.32$ & $144.11 \pm 88.93$ & $0.58+$ & 0.5607 \\
\hline CRP & $6.42 \pm 4.35$ & $5.01 \pm 3.83$ & $1.71 \dagger$ & 0.0929 \\
\hline ESR & $8.68 \pm 5.13$ & $9.09 \pm 4.71$ & $-0.42 \dagger$ & 0.6791 \\
\hline
\end{tabular}

Data are presented as mean \pm standard deviation (SD). P-values were calculated using $x^{2}$ test for categorical variables and the independent $\mathrm{t}$-test for continuous variables. $+\mathrm{t}$-value; $\neq \mathrm{X}^{2}$-value; WBC - white blood cell count; $\mathrm{N}$ - neutrophil count; $\mathrm{M}$ - monocyte count; $\mathrm{L}$ - lymphocyte count; $\mathrm{Hb}$ - hemoglobin; Plt - platelet count; RDW - red blood cell volume distribution width; PDW - platelet distribution width; MPV - mean platelet volumes; NLR - neutrophil-to-lymphocyte ratio; PLR - platelet-to-lymphocyte ratio; MLR - monocyte-to-lymphocyte ratio; CRP - C-reactive protein; ESR - erythrocyte sedimentation rate.

Table 3. Comparison of clinical characteristics and blood cell-derived parameters between immunoglobulin A (IgA) vasculitis children with renal involvement and without renal involvement

\begin{tabular}{|c|c|c|c|c|}
\hline Variable & $\begin{array}{l}\text { Renal involvement }(+) \\
\qquad(n=12)\end{array}$ & $\begin{array}{l}\text { Renal involvement }(-) \\
\qquad(n=103)\end{array}$ & Test statistic & $p$-value \\
\hline Age [years] & $9.42 \pm 2.88$ & $8.16 \pm 2.54$ & $1.60+$ & 0.1114 \\
\hline Gender (M/F) & $5 / 7$ & $51 / 52$ & $0.27 \neq$ & 0.6067 \\
\hline WBC $\left[10^{9} / \mathrm{L}\right]$ & $8.82 \pm 3.26$ & $9.57 \pm 3.66$ & $-0.68 t$ & 0.4980 \\
\hline$N\left[10^{9} / L\right]$ & $5.68 \pm 2.73$ & $6.12 \pm 3.31$ & $-0.44 \dagger$ & 0.6602 \\
\hline$M\left[10^{9} / L\right]$ & $0.43 \pm 0.23$ & $0.57 \pm 0.36$ & $-1.38+$ & 0.1691 \\
\hline $\mathrm{L}\left[10^{9} / \mathrm{L}\right]$ & $2.65 \pm 1.33$ & $2.74 \pm 1.32$ & $-0.22+$ & 0.8241 \\
\hline $\mathrm{Hb}[\mathrm{g} / \mathrm{L}]$ & $128.75 \pm 12.86$ & $125.99 \pm 11.09$ & $0.80+$ & 0.4240 \\
\hline Plt $\left[10^{9} / \mathrm{L}\right]$ & $323 \pm 87.14$ & $328.65 \pm 97.28$ & $-0.19+$ & 0.8479 \\
\hline RDW & $13.03 \pm 1.09$ & $12.76 \pm 0.88$ & $0.96+$ & 0.3349 \\
\hline PDW & $14.07 \pm 2.19$ & $12.92 \pm 2.7$ & $1.42 \dagger$ & 0.1583 \\
\hline MPV & $10.33 \pm 1.4$ & $10.1 \pm 1.32$ & $0.55+$ & 0.5812 \\
\hline NLR & $2.62 \pm 1.95$ & $2.85 \pm 2.47$ & $-0.31+$ & 0.7550 \\
\hline MLR & $0.2 \pm 0.14$ & $0.23 \pm 0.14$ & $-0.71+$ & 0.4803 \\
\hline PLR & $143.98 \pm 61.63$ & $147.45 \pm 86.65$ & $-0.13+$ & 0.8929 \\
\hline CRP & $4.63 \pm 3.86$ & $5.51 \pm 4.09$ & $-0.67+$ & 0.4991 \\
\hline ESR & $8.58 \pm 4.12$ & $9.01 \pm 4.91$ & $-0.29+$ & 0.7732 \\
\hline
\end{tabular}

Data are presented as mean \pm standard deviation (SD). P-values were calculated using $x^{2}$ test for categorical variables and the independent t-test for continuous variables. + t-value; $\neq X^{2}$-value; WBC - white blood cell count; $N$ - neutrophil count; $M$ - monocyte count; $L$ - lymphocyte count; $\mathrm{Hb}$ - hemoglobin; Plt - platelet count; RDW - red blood cell volume distribution width; PDW - platelet distribution width; MPV - mean platelet volumes; NLR - neutrophil-to-lymphocyte ratio; PLR - platelet-to-lymphocyte ratio; MLR - monocyte-to-lymphocyte ratio; CRP - C-reactive protein; ESR - erythrocyte sedimentation rate. 


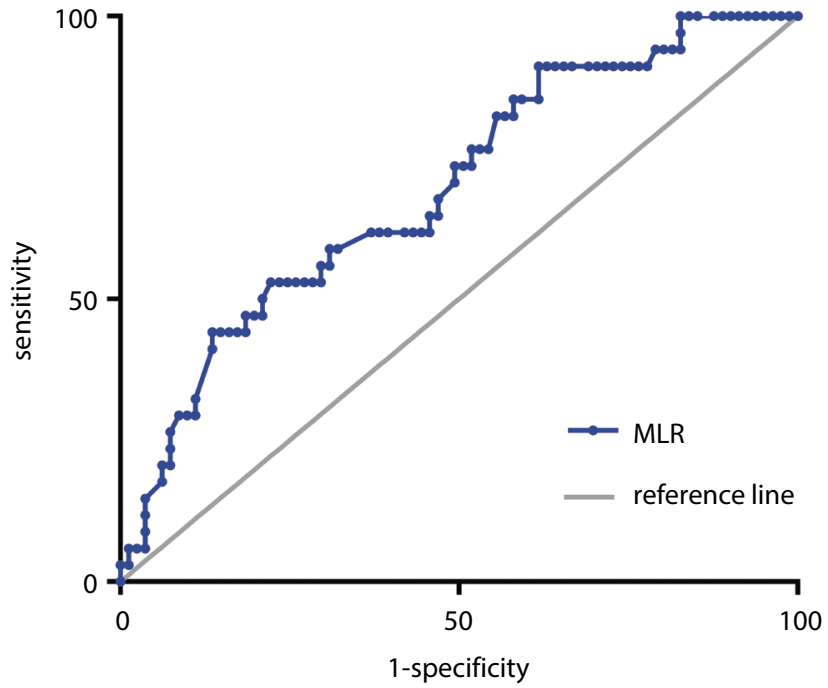

Fig. 1. Receiver operating characteristic curves of the monocyte-tolymphocyte ratio (MLR) for children with immunoglobulin A ( $\lg A)$ vasculitis with gastrointestinal system involvement inexpensive and practical biomarkers were investigated. The current study demonstrated that children with IgA vasculitis with GS involvement showed higher neutrophil counts and MLR and NLR values. Moreover, elevated MLR was found to be a risk factor for GS involvement in children with IgA vasculitis, and ROC analysis indicated that MLR could be a predictive parameter of GS involvement in IgA vasculitis. To the best of our knowledge, this study is the first to comprehensively assess new blood cell-derived inflammatory biomarkers, especially MLR, in relation to GS involvement and disease course in IgA vasculitis in children.

In the current study, we found that the incidence of GS involvement in the current cohort is $29.56 \%(\mathrm{n}=34)$, and $10.43 \%(\mathrm{n}=12)$ for renal involvement. According to a cross-section study conducted in Turkey, clinical manifestations with gastrointestinal symptoms and renal involvement among children with IgA vasculitis appeared in, respectively, $56 \%$ and $29.8 \%$ of cases. ${ }^{20}$ Similarly,

Table 4. Comparison of clinical characteristics and blood cell-derived parameters between immunoglobulin A (IgA) vasculitis children with recurrence and without recurrence

\begin{tabular}{|c|c|c|c|c|}
\hline Variable & $\begin{array}{l}\text { Recurrence }(+) \\
\quad(n=22)\end{array}$ & $\begin{array}{l}\text { Recurrence }(-) \\
\qquad(n=93)\end{array}$ & Test statistic & $p$-value \\
\hline Age [years] & $8.05 \pm 2.64$ & $8.34 \pm 2.59$ & $-0.48 \dagger$ & 0.6295 \\
\hline Gender (M/F) & $12 / 10$ & $47 / 46$ & $0.11 \neq$ & 0.7352 \\
\hline WBC $\left[10^{9} / L\right]$ & $8.77 \pm 3.09$ & $9.66 \pm 3.72$ & $-1.04 \dagger$ & 0.2995 \\
\hline$N\left[10^{9} / L\right]$ & $5.47 \pm 2.32$ & $6.22 \pm 3.43$ & $-0.97 \dagger$ & 0.3351 \\
\hline$M\left[10^{9} / L\right]$ & $0.56 \pm 0.43$ & $0.56 \pm 0.33$ & $0.04 \dagger$ & 0.9685 \\
\hline $\mathrm{L}\left[10^{9} / \mathrm{L}\right]$ & $2.6 \pm 1.12$ & $2.76 \pm 1.36$ & $-0.53 \dagger$ & 0.5976 \\
\hline $\mathrm{Hb}[\mathrm{g} / \mathrm{L}]$ & $126.55 \pm 15.35$ & $126.22 \pm 10.16$ & $0.12+$ & 0.9021 \\
\hline Plt $\left[10^{9} / \mathrm{L}\right]$ & $316.55 \pm 106.3$ & $330.78 \pm 93.73$ & $-0.62 \dagger$ & 0.5336 \\
\hline RDW & $12.7 \pm 0.71$ & $12.81 \pm 0.94$ & $-0.48+$ & 0.6314 \\
\hline PDW & $14.25 \pm 2.63$ & $12.75 \pm 2.61$ & $2.42 \dagger$ & 0.0170 \\
\hline MPV & $10.1 \pm 1.53$ & $10.13 \pm 1.28$ & $-0.08 t$ & 0.9382 \\
\hline NLR & $2.54 \pm 1.72$ & $2.9 \pm 2.55$ & $-0.63+$ & 0.5309 \\
\hline MLR & $0.22 \pm 0.10$ & $0.23 \pm 0.15$ & $-0.29 t$ & 0.7749 \\
\hline PLR & $141.38 \pm 69.41$ & $148.44 \pm 87.58$ & $-0.35 \dagger$ & 0.7250 \\
\hline CRP & $4.48 \pm 3.57$ & $5.64 \pm 4.15$ & $-1.18+$ & 0.2397 \\
\hline ESR & $9.55 \pm 5.53$ & $8.83 \pm 4.66$ & $0.63+$ & 0.5324 \\
\hline
\end{tabular}

Data are presented as mean \pm standard deviation (SD). P-values were calculated using $x^{2}$ test for categorical variables and the independent t-test for continuous variables. $+\mathrm{t}$-value; $\neq \mathrm{X}^{2}$-value; WBC - white blood cell count; $\mathrm{N}$ - neutrophil count; $M$ - monocyte count; $L$ - lymphocyte count; $\mathrm{Hb}$ - hemoglobin; PIt - platelet count; RDW - red blood cell volume distribution width; PDW - platelet distribution width; MPV - mean platelet volumes; NLR - neutrophil-to-lymphocyte ratio; PLR - platelet-to-lymphocyte ratio; MLR - monocyte-to-lymphocyte ratio; CRP - C-reactive protein; ESR - erythrocyte sedimentation rate.

Table 5. Multivariate analysis for immunoglobulin A ( $\mathrm{lgA}$ ) vasculitis children combined with gastrointestinal system involvement

\begin{tabular}{|c|c|c|c|c|c|c|c|c|}
\hline \multirow{2}{*}{ Parameter } & \multirow{2}{*}{ Coefficient } & \multirow{2}{*}{ SE } & \multirow{2}{*}{ Wald $x^{2}$ value } & \multirow{2}{*}{ df } & \multirow{2}{*}{$p$-value } & \multirow{2}{*}{ OR } & \multicolumn{2}{|c|}{$95 \% \mathrm{Cl}$} \\
\hline & & & & & & & lower & upper \\
\hline N & 0.03 & 0.09 & 0.11 & 1.00 & 0.73 & 1.03 & 0.86 & 1.23 \\
\hline MLR & 4.49 & 1.96 & 5.22 & 1.00 & 0.02 & 89.06 & 1.89 & 4190.12 \\
\hline NLR & 0.09 & 0.13 & 0.48 & 1.00 & 0.49 & 1.09 & 0.85 & 1.40 \\
\hline
\end{tabular}

N - neutrophil count; NLR - neutrophil-to-lymphocyte ratio; MLR - monocyte-to-lymphocyte ratio; SE - standard error; OR - odds ratio; 95\% Cl - 95\% confidence interval; df - degrees of freedom. 
gastrointestinal symptoms and renal kidney involvement among the children with IgA vasculitis in Poland were found in, respectively, $64.8 \%$ and $22.5 \%$ of cases. ${ }^{25}$ In addition, GS involvement was found to be $46.1 \%$ in Korea. ${ }^{12}$ However, according to a retrospective analysis conducted in China, the incidence of GS symptoms and kidney involvement was $28.78 \%$ and $13.33 \%$, respectively, among children with IgA vasculitis, which is similar to our study and much lower than in other studies. We postulate the divergence of GS and kidney involvement may relate to racial and regional differences.

Blood cell count-derived inflammatory parameters, including NLR and PLR, are now recognized as novel biomarkers of chronic subclinical inflammation in diabetes mellitus, ${ }^{21}$ coronary artery disease ${ }^{8}$ and cancers. ${ }^{22}$ In this study, we demonstrated that the NLR but not PLR value was significantly increased in children with IgA vasculitis with GS involvement compared to those without GS involvement, which is consistent with the study by Karadağ et al. ${ }^{19}$ Furthermore, it was reported that elevated NLR and PLR may be relevant markers for predicting GS bleeding ${ }^{23}$ and renal organ involvement ${ }^{24,25}$ in children with IgA vasculitis. However, there was no relationship between NLR and PLR values and renal or GS involvement in the current study. This inconsistency may be due to different degrees of GS and kidney complication among the included participants, which suggests the predictive value of NLR and PLR in children with IgA vasculitis may depend on the severity of the disease, and further extensive multicenter studies are needed to clarify this issue.

The MLR has been demonstrated as a novel hematological parameter in several medical fields. Recently, Suszek et al. demonstrated that MLR is an effective marker of systemic lupus erythematosus activity, ${ }^{26}$ and its value could also be used to identify disease activity of Takayasu arteritis ${ }^{27}$ and rheumatoid arthritis. ${ }^{28}$ Similarly, in the current study, MLR values were higher in children with IgA vasculitis with GS involvement than in those without GS involvement. Interestingly, MLR is the sole risk factor that predicted GS involvement in children with IgA vasculitis children. To our knowledge, this is the first study to reveal the predictive value of MLR in children with IgA vasculitis.

The elevated MLR level may result from increased monocyte counts or decreased lymphocyte counts. Monocytes are an essential source of pro-inflammatory mediators during various kinds of vasculitis. ${ }^{29-31}$ It was reported that monocytes were the predominant cell type infiltrating glomeruli in IgA nephritis, ${ }^{31}$ which indicates the monocyte count may reflect the development of IgA vasculitis. On the other hand, lymphocytes have been shown to play a broader role in modulating the inflammatory response at each phase of the vasculitis, and low lymphocyte count has been associated with IgA vasculitis progression in rat and rabbit models. ${ }^{32}$ Local activation of the immune system or inflammation can increase lymphocyte apoptosis, ${ }^{33}$ resulting in lower lymphocyte counts. Therefore, higher monocyte counts and/or lower lymphocyte counts may reflect an active role due to their immunomodulatory, pro-inflammatory effects on vasculitis. Thus, the MLR integrated with monocytes and lymphocytes provided a more reliable index to evaluate IgA vasculitis inflammation than either variable alone.

\section{Limitations}

This study has some limitations. Firstly, the sample size of this study is not large, and it is a single-center study. Secondly, the predictive value of MLR is probably more reliable than the commonly used laboratory inflammatory indicators CRP and ESR in evaluating GS involvement of IgA vasculitis in the current study; however, the predictive value of blood cell-derived inflammatory parameters cannot be compared with other inflammatory markers, including interleukin (IL)-6 and tumor necrosis factor alpha (TNF- $\alpha),{ }^{34-36}$ due to the retrospective design and lack of related data. Another limitation was the lack of dynamic changes in hematological parameters at IgA vasculitis diagnosis and after long-term follow-up, which could provide more information about the relationships between these hematological parameter values and disease course.

\section{Conclusions}

This study showed that neutrophil count and NLR and MLR levels were found to be significantly higher in the children with IgA vasculitis with GS involvement, and elevated MLR was an independent risk factor in predicting GS involvement in children with IgA vasculitis. Therefore, monitoring blood MLR values could provide an early indicator to clinicians regarding GS involvement and disease severity in children with IgA vasculitis.

\section{ORCID iDs}

Yuan Yuan (10) https://orcid.org/0000-0002-0958-4757 Jun Liu (i) https://orcid.org/0000-0001-8148-9175 Yongxin Zhou (1) https://orcid.org/0000-0003-3119-9721 Xufang Du (i) https://orcid.org/0000-0002-9711-0198 Qian Chen (1) https://orcid.org/0000-0001-7780-0573 Jiongying Zhou (1) https://orcid.org/0000-0002-8651-3071 Miao Hou (ib https://orcid.org/0000-0002-9931-5146

\section{References}

1. Dyga K, Szczepanska M. IgA vasculitis with nephritis in children. Adv Clin Exp Med. 2020;29(4):513-519. doi:10.17219/acem/112566

2. Leung A, Barankin B, Leong KF. Henoch-Schönlein purpura in children: An updated review. Curr Pediatr Rev. 2020;16(4):265-276. doi:10.2174/1573396316666200508104708

3. Calvo-Rio V, Hernandez JL, Ortiz-Sanjuan F, et al. Relapses in patients with Henoch-Schönlein purpura: Analysis of 417 patients from a single center. Medicine (Baltimore). 2016;95(28):e4217. doi:10.1097/MD. 0000000000004217

4. Ozen S, Sag E. Childhood vasculitis. Rheumatology (Oxford). 2020; 59(Suppl 3):iii95-iii100. doi:10.1093/rheumatology/kez599

5. Cho $\mathrm{JH}, \mathrm{ChoHJ}$, Lee HY, et al. Neutrophil-lymphocyte ratio in patients with acute heart failure predicts in-hospital and long-term mortality. J Clin Med. 2020;9(2):557. doi:10.3390/jcm9020557 
6. Masoumi M, Shadmanfar S, Davatchi F, et al. Correlation of clinical signs and symptoms of Behcet's disease with mean platelet volume (MPV) and red cell distribution width (RDW). Orphanet J Rare Dis. 2020;15(1):297. doi:10.1186/s13023-020-01588-1

7. Li X, Shen J, Lu Z, et al. High neutrophil-to-lymphocyte ratio is associated with increased carotid artery intima-media thickness in type 2 diabetes. J Diabetes Investig. 2017;8(1):101-107. doi:10.1111/jdi.12541

8. Haybar H, Pezeshki S, Saki N. Evaluation of complete blood count parameters in cardiovascular diseases: An early indicator of prognosis? Exp Mol Pathol. 2019;110:104267. doi:10.1016/j.yexmp.2019.104267

9. Mertoglu C, Gunay M. Neutrophil-lymphocyte ratio and plateletlymphocyte ratio as useful predictive markers of prediabetes and diabetes mellitus. Diabetes Metab Syndr. 2017;11(Suppl 1):S127-S131. doi:10.1016/j.dsx.2016.12.021

10. Paliogiannis P, Satta R, Deligia G, et al. Associations between the neutrophil-to-lymphocyte and the platelet-to-lymphocyte ratios and the presence and severity of psoriasis: A systematic review and meta-analysis. Clin Exp Med. 2019;19(1):37-45. doi:10.1007/s10238018-0538-x

11. Han D, Zhang J, Zhao J, et al. Platelet-to-lymphocyte ratio is an independent predictor of chemoradiotherapy-related esophageal fistula in esophageal cancer patients. Ann Transl Med. 2020;8(18):1163. doi:10.21037/atm-20-4053

12. Hong $\mathrm{SH}$, Kim CJ, Yang EM. Neutrophil-to-lymphocyte ratio to predict gastrointestinal bleeding in Henoch-Schönlein purpura. Pediatr Int. 2018;60(9):791-795. doi:10.1111/ped.13652

13. Jakubowska K, Koda M, Grudzinska M, Kanczuga-Koda L, Famulski W. Monocyte-to-lymphocyte ratio as a prognostic factor in peripheral whole blood samples of colorectal cancer patients. World J Gastroenterol. 2020;26(31):4639-4655. doi:10.3748/wjg.v26.i31.4639

14. Li X, Wu J, Mao W. Evaluation of the neutrophil-to-lymphocyte ratio monocyte-to-lymphocyte ratio, and red cell distribution width for the prediction of prognosis of patients with hepatitis $B$ virus-related decompensated cirrhosis. J Clin Lab Anal. 2020;34(11):e23478. doi:10. 1002/jcla.23478

15. Hu YX, Xu XX, Shao $Y$, et al. The prognostic value of lymphocyteto-monocyte ratio in retinopathy of prematurity. Int J Ophthalmol. 2017;10(11):1716-1721. doi:10.18240/ijo.2017.11.13

16. Jennette JC, Falk RJ, Bacon PA, et al. 2012 revised International Chapel Hill Consensus Conference Nomenclature of Vasculitides. Arthritis Rheum. 2013;65(1):1-11. doi:10.1002/art.37715

17. Ruperto N, Ozen S, Pistorio A et, al. EULAR/PRINTO/PRES criteria for Henoch-Schönlein purpura, childhood polyarteritis nodosa, childhood Wegener granulomatosis and childhood Takayasu arteritis: Ankara 2008. Part I: Overall methodology and clinical characterisation. Ann Rheum Dis. 2010;69(5):790-797. doi:10.1136/ard.2009.116624

18. Paek EY, Yi DY, Kang B, Choe BH. Fecal calprotectin as a marker of gastrointestinal involvement in pediatric Henoch-Schönlein purpura patients: A retrospective analysis. BMC Pediatr. 2020;20(1):374. doi:10.1186/s12887-020-02263-x

19. Karadag SG, Cakmak F, Cil B, et al. The relevance of practical laboratory markers in predicting gastrointestinal and renal involvement in children with Henoch-Schönlein purpura. Postgrad Med. 2021;133(3):272-277. doi:10.1080/00325481.2020.1807161

20. Ekinci R, Balci S, Melek E, et al. Clinical manifestations and outcomes of 420 children with Henoch-Schönlein purpura from a single referral center from Turkey: A three-year experience. Mod Rheumatol. 2020;30(6):1039-1046. doi:10.1080/14397595.2019.1687074

21. Demirtas L, Degirmenci H, Akbas EM, et al. Association of hematological indicies with diabetes, impaired glucose regulation and microvascular complications of diabetes. Int JClin Exp Med. 2015;8(7): 11420-11427. PMID:26379958
22. de Jong MC, Mihai R, Khan S. Neutrophil-to-lymphocyte ratio (NLR) and platelet-to-lymphocyte ratio (PLR) as possible prognostic markers for patients undergoing resection of adrenocortical carcinoma. World J Surg. 2020;45(3):754-764. doi:10.1007/s00268-020-05868-6

23. Gayret OB, Erol M, Tekin NH. The relationship of neutrophil-lymphocyte ratio and platelet-lymphocyte ratio with gastrointestinal bleeding in Henoch-Schönlein purpura. Iran J Pediatr. 2016;26(5):e8191. doi:10.5812/ijp.8191

24. Ozdemir ZC, Cetin N, Kar YD, et al. Hemotologic indices for predicting internal organ involvement in Henoch-Schönlein purpura ( IgA vasculitis). J Pediatr Hematol Oncol. 2020;42(1):e46-e49. doi:10.1097/ MPH.0000000000001571

25. Jaszczura M, Gora A, Grzywna-RozenekE, Barc-Czarnecka M, Machura E. Analysis of neutrophil to lymphocyte ratio, platelet to lymphocyte ratio and mean platelet volume to platelet count ratio in children with acute stage of immunoglobulin A vasculitis and assessment of their suitability for predicting the course of the disease. Rheumatol Int. 2019;39(5):869-878. doi:10.1007/s00296-019-04274-z

26. Suszek D, Gorak A, Majdan M. Differential approach to peripheral blood cell ratios in patients with systemic lupus erythematosus and various manifestations. Rheumatol Int. 2020;40(10):1625-1629. doi:10.1007/s00296-020-04669-3

27. Seringec AN, Yildirim CG, Gogebakan $\mathrm{H}$, Acipayam C. The C-reactive protein/albumin ratio and complete blood count parameters as indicators of disease activity in patients with Takayasu arteritis. Med Sci Monit. 2019;25:1401-1409. doi:10.12659/MSM.912495

28. Du J, Chen S, Shi J, et al. The association between the lymphocyte-monocyte ratio and disease activity in rheumatoid arthritis. Clin Rheumatol. 2017;36(12):2689-2695. doi:10.1007/s10067-017-3815-2

29. Matsumoto K, Suzuki K, Yoshimoto K, et al. Longitudinal immune cell monitoring identified CD14(++) CD16(+) intermediate monocyte as a marker of relapse in patients with ANCA-associated vasculitis. Arthritis Res Ther. 2020;22(1):145. doi:10.1186/s13075-020-02234-8

30. Verweij SL, Duivenvoorden R, Stiekema L, et al. CCR2 expression on circulating monocytes is associated with arterial wall inflammation assessed by 18F-FDG PET/CT in patients at risk for cardiovascular disease. Cardiovasc Res. 2018;114(3):468-475. doi:10.1093/cvr/cvx224

31. Luo $Y$, Yang J, Zhang $C$, et al. Upregulation of miR-27a promotes monocyte-mediated inflammatory responses in Kawasaki disease by inhibiting function of B10 cells. J Leukoc Biol. 2020;107(1):133-144. doi:10.1002/JLB.5A0919-075RR

32. Li $Y$, Feng $X$, Huang $L$, et al. Hematologic and immunological characteristics of Henoch-Schönlein purpura in rat and rabbit models induced with ovalbumin based on type III hypersensitivity. Sci Rep. 2015;5:8862. doi:10.1038/srep08862

33. Ozaltin F, Besbas N, Uckan D, et al. The role of apoptosis in childhood Henoch-Schönlein purpura. Clin Rheumatol. 2003;22(4-5):265-267. doi:10.1007/s10067-003-0718-1

34. Chan $\mathrm{H}$, Tang YL, Lv XH, et al. Risk factors associated with renal involvement in childhood Henoch-Schönlein purpura: A meta-analysis. PLoS One. 2016;11(11):e0167346. doi:10.1371/journal.pone.0167346

35. Sugiyama $M$, Wada $Y$, Kanazawa $N$, et al. A cross-sectional analysis of clinicopathologic similarities and differences between HenochSchönlein purpura nephritis and IgA nephropathy. PLOS One. 2020; 15(4):e0232194. doi:10.1371/journal.pone.0232194

36. Kuret $\mathrm{T}$, Lakota $\mathrm{K}$, Zigon $\mathrm{P}$, et al. Insight into inflammatory cell and cytokine profiles in adult IgA vasculitis. Clin Rheumatol. 2019;38(2): 331-338. doi:10.1007/s10067-018-4234-8 